\title{
Cannabis use and the risk of tuberculosis: a systematic review
}

\author{
Clare E. French ${ }^{1,2+}$, Caroline M. Coope ${ }^{2,3^{*} \dagger}$, Luke A. McGuinness ${ }^{1,2}$, Charles R. Beck ${ }^{2,3}$, Sophie Newitt ${ }^{4}$, \\ Lauren Ahyow ${ }^{5}$, Matt Hickman ${ }^{1,2}$ and Isabel Oliver ${ }^{2,3}$
}

\begin{abstract}
Background: Cannabis has been identified as a possible risk factor in some tuberculosis (TB) outbreaks. As the most widely used (largely) illegal substance in Western countries this may be an important public health concern. We aim to systematically review the evidence on the association between cannabis use and TB (latent infection and active disease) to inform ongoing and future TB prevention and control strategies.

Methods: We conducted a systematic review. We searched Ovid Medline, Embase and Psyclnfo, together with the World Health Organization website and Google Scholar, for all years to January 2018. Reference lists and conference abstracts were hand-searched, a forward citation search was conducted on the Web of Science, and experts were contacted. Two authors independently screened studies for inclusion, extracted data and assessed risk of bias using an adapted version of ROBINS-I ("Risk of Bias in Non-randomised Studies - of Interventions"). Data were narratively synthesised.
\end{abstract}

Results: Of 377 records identified, 11 studies were eligible. Study designs were heterogeneous. Six studies utilised a relevant comparator group. Four of these investigated the association between cannabis use and latent TB infection; all provided some evidence of an association, although only two of these had adjusted for confounders. The remaining two comparator studies investigated the association between cannabis use and active TB disease; neither found evidence of an association after adjusting for confounding. All six studies were at "Serious" risk of bias. The five studies which did not utilise a relevant comparator group were all indicative of TB outbreaks occurring among cannabis users, but the quality of the evidence was very weak.

Conclusions: Evidence for an association between cannabis use and TB acquisition is weak. The topic warrants further robust primary research including the collection of consistent and accurate exposure information, including cannabis use practices, dose and frequency, and adjustment for confounders.

Keywords: Tuberculosis, Cannabis, Systematic review, Evidence synthesis

\section{Background}

It is estimated that 1.7 billion people are infected with Mycobacterium tuberculosis (TB) globally of which 5 to $15 \%$ will develop active TB disease, depending on coexisting risk factors such as under-nutrition, diabetes, HIV (human immunodeficiency virus) infection, smoking and

\footnotetext{
* Correspondence: caroline.coope@bristol.ac.uk

Clare E French and Caroline M Coope are Co-first authors

${ }^{+}$Clare E. French and Caroline M. Coope contributed equally to this work.

${ }^{2}$ National Institute for Health Research Health Protection Unit in Evaluation of Interventions, Bristol Medical School, University of Bristol, Bristol BS8 2PS, UK

${ }^{3}$ Field Service South West, National Infection Service, Public Health England, Bristol BS1 6EH, UK

Full list of author information is available at the end of the article
}

alcohol consumption [1]. In high-income countries TB has most commonly occurred in marginalised populations such as migrants, the homeless, people who inject drugs and prisoners [2-4]. With the introduction of whole genome sequencing in TB outbreak investigation the accurate linkage of cases, even prior to epidemiological linkage [5], has facilitated the identification of previously unrecognised risk factors for transmission. These novel risk factors, such as cannabis use, may be important to inform outbreak investigations and control efforts and need to be better understood.

Cannabis is estimated to be the most widely used (largely) illegal substance in Western countries including

(c) The Author(s). 2019 Open Access This article is distributed under the terms of the Creative Commons Attribution 4.0 International License (http://creativecommons.org/licenses/by/4.0/), which permits unrestricted use, distribution, and reproduction in any medium, provided you give appropriate credit to the original author(s) and the source, provide a link to the Creative Commons license, and indicate if changes were made. The Creative Commons Public Domain Dedication waiver (http://creativecommons.org/publicdomain/zero/1.0/) applies to the data made available in this article, unless otherwise stated. 
Europe [6], North America [7] and Australia [8]. Frequent cannabis use has been reported among cases in previous TB outbreaks $[9,10]$, and recently in an outbreak of pulmonary TB in the United Kingdom (UK) in which $26 \%$ of those with latent TB infection (LTBI) and 67\% with TB disease reported cannabis smoking (Lauren Ahyow, Public Health England - personal communication). Given the prevalence of cannabis use, and the increasing potency available [6], there is a pressing need to understand whether there is an association between cannabis use and TB - a potentially significant public health concern.

The method used to inhale cannabis could be important in TB transmission and links have been found with sharing a cannabis water pipe or 'bong' [11] and 'shotgunning' (the practice of inhaling smoke and then exhaling it into another individual's mouth) [12]. These behaviours may offer an environment for the efficient transmission of $\mathrm{TB}$, such as prolonged exposure and close proximity to a case. Evidence supports the biological plausibility of an association as heavy cannabis smoking has been associated with chronic bronchitis symptoms and large airway inflammation which may lower the lungs natural defences against infection [13]. Additionally, there is evidence that tobacco smoking, often adjunct to cannabis use, is associated with TB infection and disease [14] and may increase the risk of disease by as much as 2.5 times [15].

To our knowledge, evidence on the possible association between cannabis use and TB infection and disease has not been systematically reviewed. We aim to address this gap to inform ongoing and future TB prevention and control strategies.

\section{Methods}

This review is reported in accordance with the Preferred Reporting Items for Systematic Reviews and MetaAnalyses (PRISMA) [16]. The review protocol can be obtained by request from the corresponding author.

\section{Eligibility criteria}

All types of primary epidemiological studies (e.g. descriptive studies, outbreak reports, cohort studies, case-control studies) were eligible for inclusion. The review question was structured using a PECO framework as follows:Population: adults aged $\geq 16$ years; Exposure: cannabis use by any means; Comparator: any e.g. no reported cannabis use, no comparator; Outcome: active TB disease affecting any clinical site (pulmonary or extra-pulmonary) or latent infection e.g. assessed by Tuberculin Skin Testing [TST] ('Mantoux' test) or an interferon-gamma release assay. No restriction was placed on the type/availability of a specific comparator group in order that the complete body of evidence could be reviewed.

\section{Search strategy}

Electronic searches were conducted in Ovid Medline, Embase and PsycInfo, from the inception of each database to 17 January 2018. Subject Headings (MeSH, EMTREE, APA) and free-text key words were used (see Additional file 1 for search terms). No restriction was placed on language or publication status. The World Health Organization website and Google Scholar were searched. We also checked abstract lists for the annual Union World Conference on Lung Health for the years 2015-2017. Reference lists of eligible studies were handsearched, and a forward citation search was conducted in Web of Science to help identify follow-up studies or new research citing any of the study reports included in the review. Experts in the field were contacted to identify any further published or unpublished studies. Records were stored and managed in EndNote X9.

\section{Study selection}

Two authors screened studies for eligibility and agreed the final list for inclusion. Reasons for exclusion of studies that reached the full-text screening stage were documented.

\section{Data extraction and synthesis}

Data from eligible studies was double extracted by two reviewers (CEF and CMC) using a pre-defined form, and cross-checked. Key data items (as listed in Table 1) were extracted and narratively synthesized. Summary measures reported varied; we extracted odds ratios (e.g. comparing the odds of $\mathrm{TB}$ in those who reported using cannabis vs. those who did not) whenever documented. Studies which utilised a relevant comparator group are reported separately from those which did not. Given the substantial heterogeneity between studies it was not appropriate to perform a meta-analysis.

\section{Risk of bias assessments}

Risk of bias was assessed for studies which utilised a comparator group. Each distinct result from these studies was assessed across six risk of bias domains using an adapted version of the ROBINS-I tool ("Risk of Bias in Non-randomised Studies - of Interventions"), [17] informed by the preliminary ROBINS-E tool ("Risk of Bias In Non-randomised Studies - of Exposure") [18]. Although ROBINS-I is designed to assess risk of bias in intervention studies many of the domains are relevant to non-randomised studies of exposures. Summary level judgements were made for each domain and were used to inform an overall risk of bias judgement (see Additional file 2 for guidance used to make domain level risk of bias judgements). 


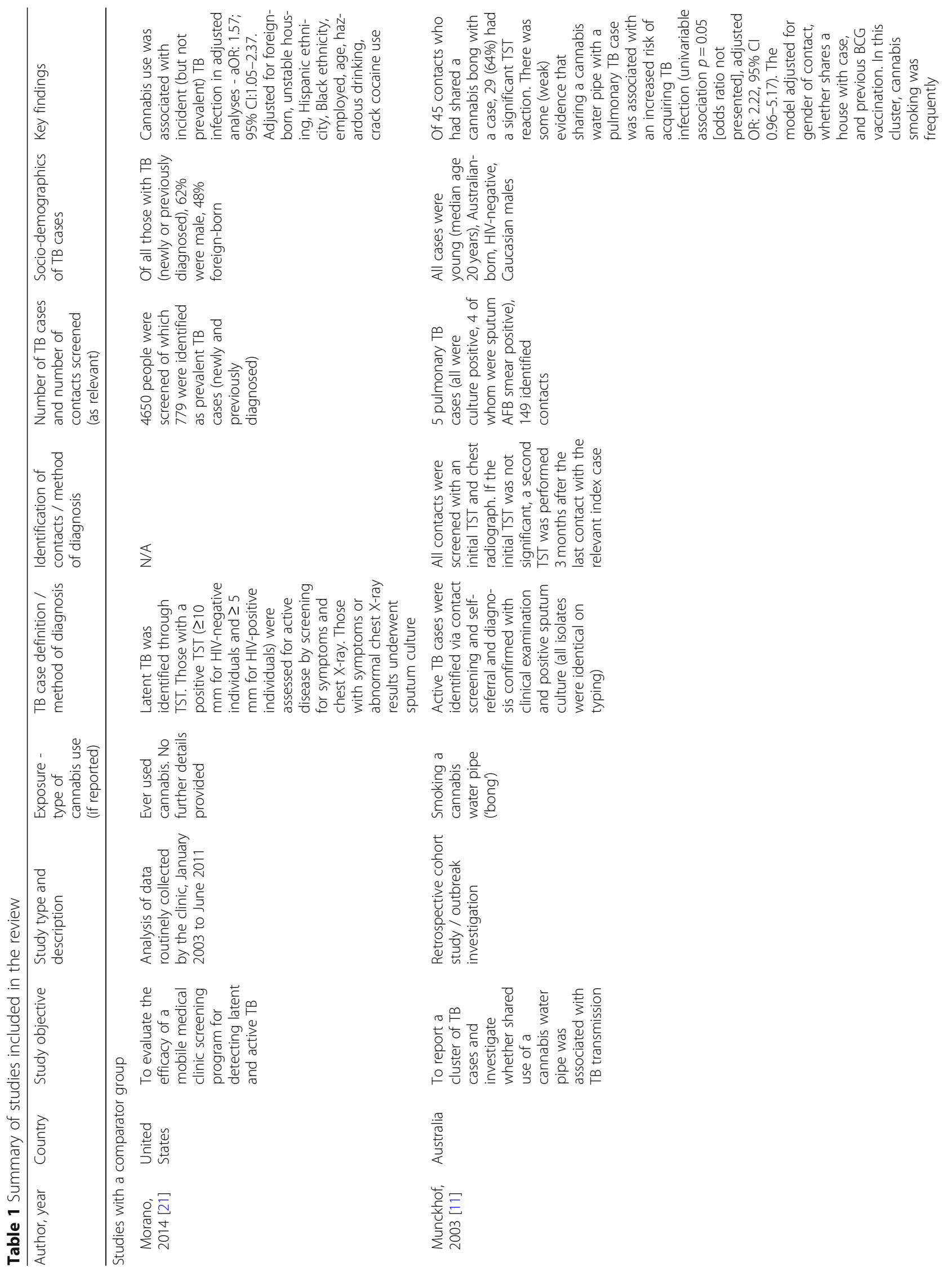




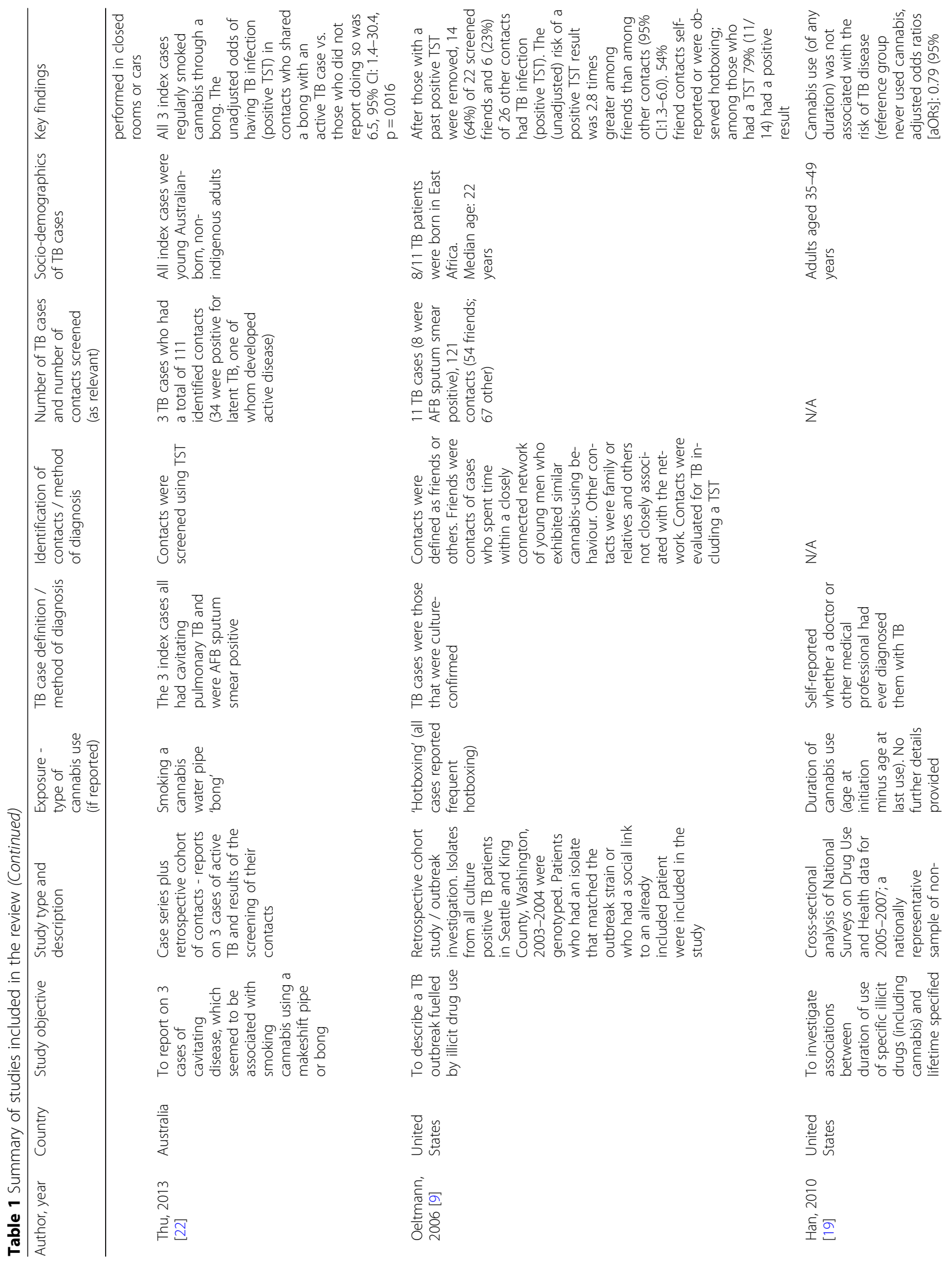




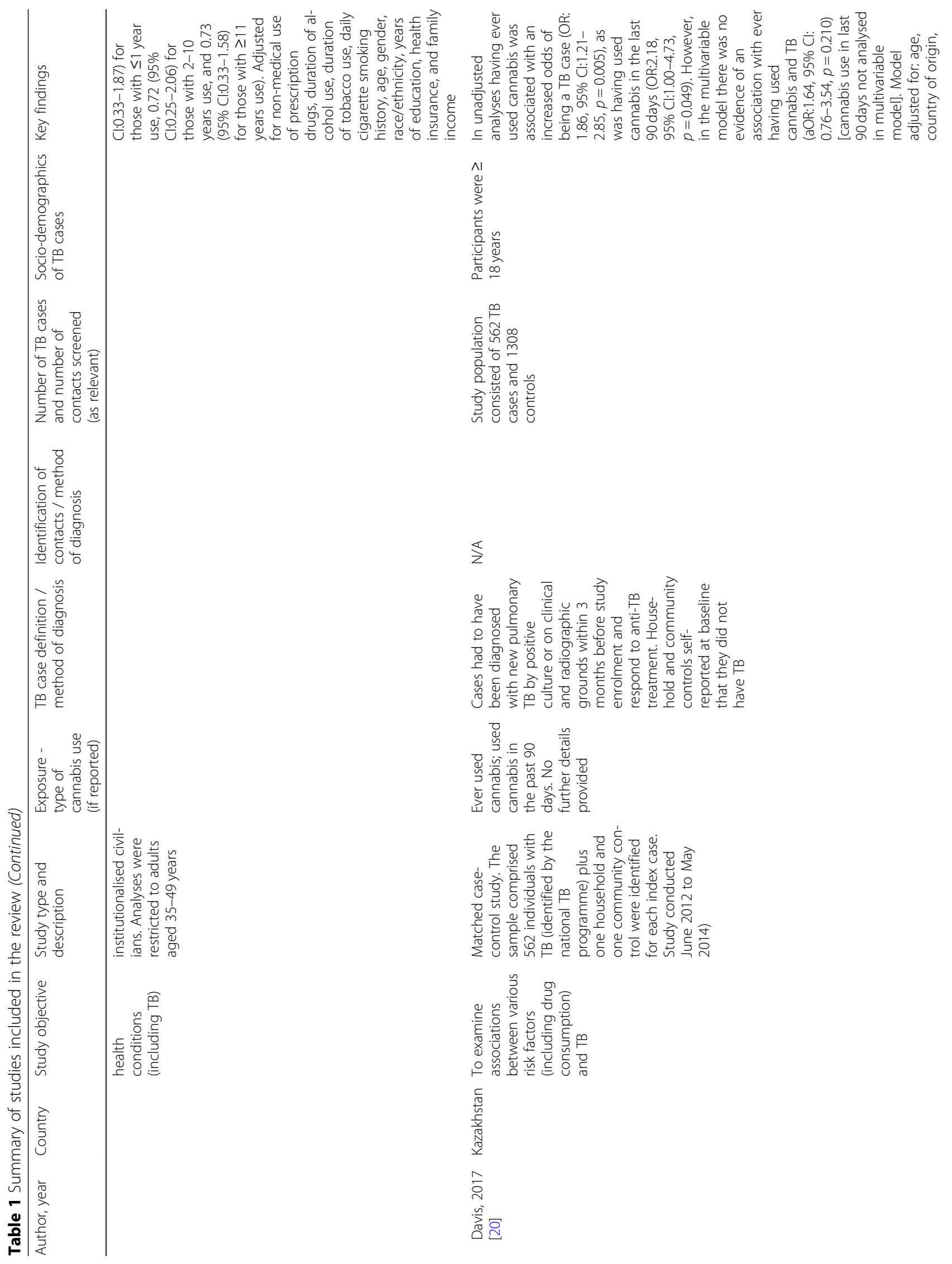




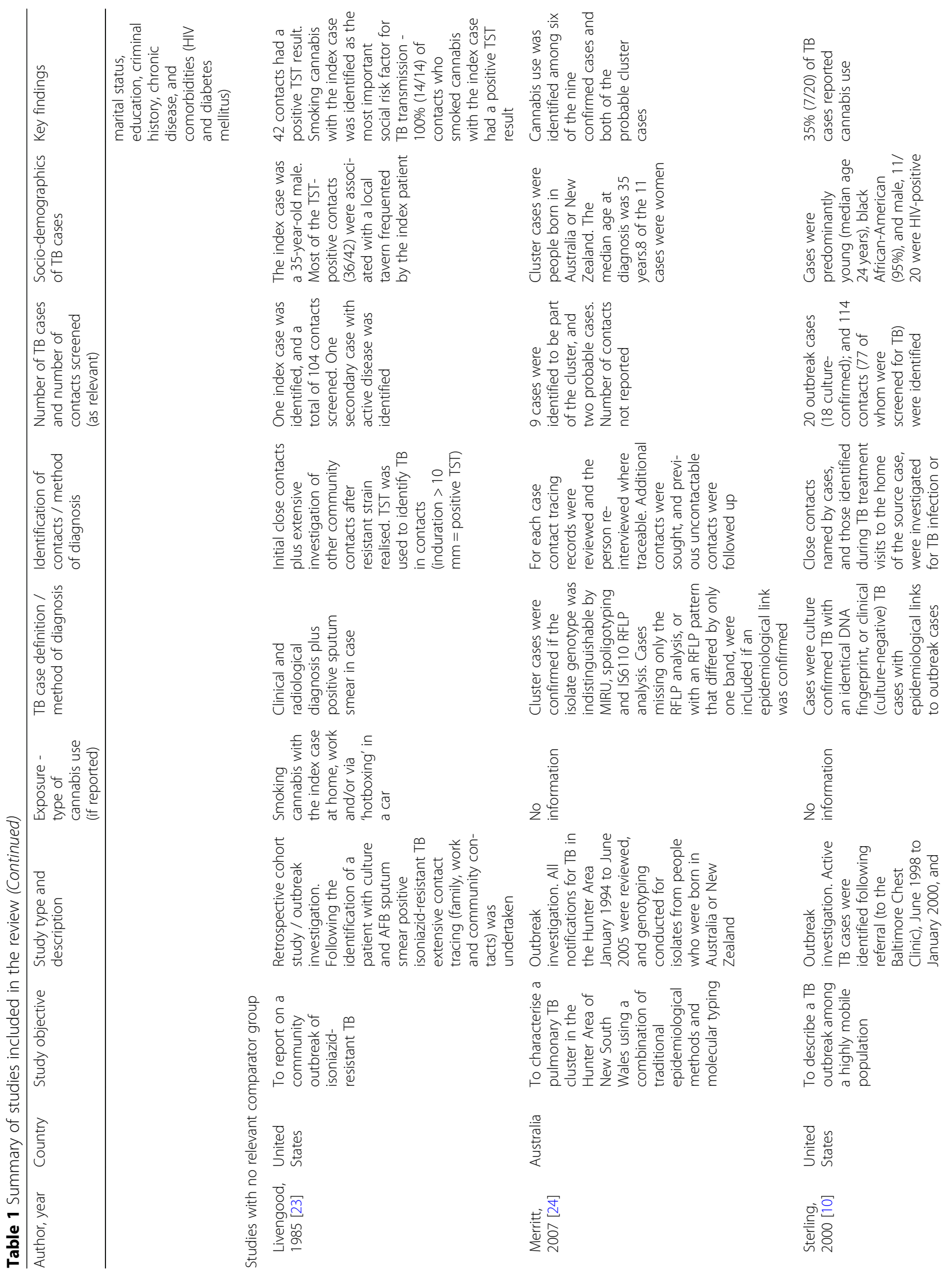




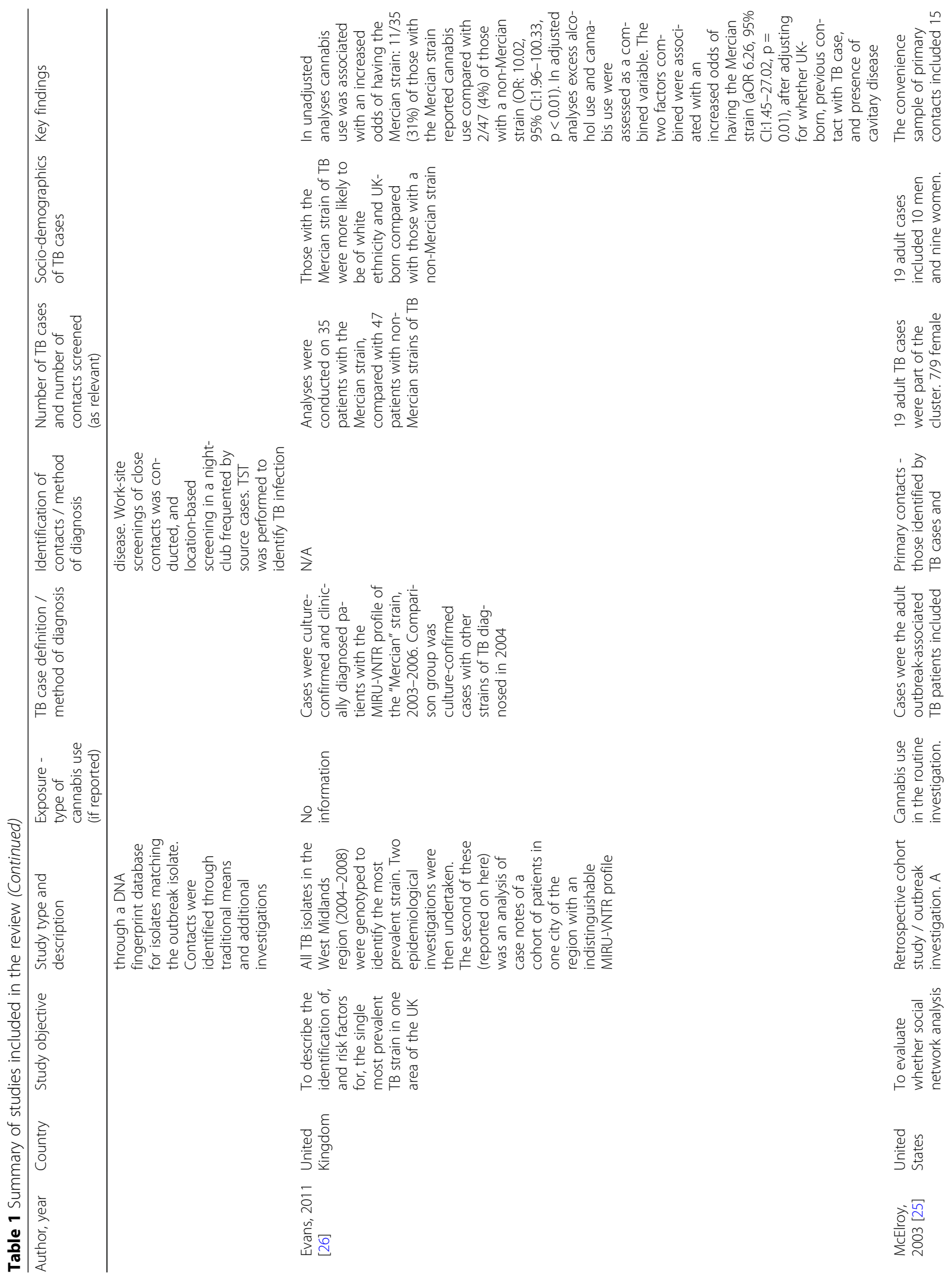




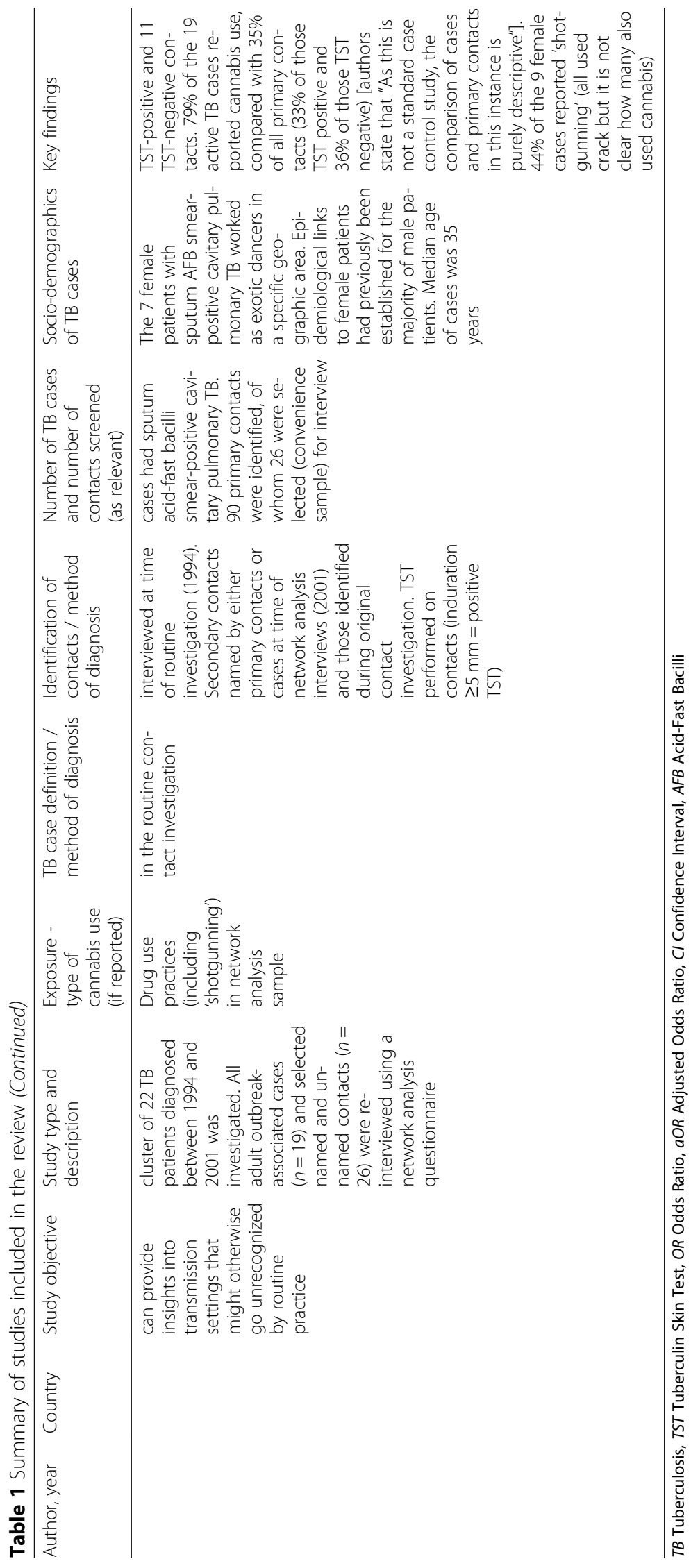




\section{Results}

\section{Description of studies}

After removal of duplicates, the initial electronic searches identified 373 potentially eligible records, with four additional eligible records identified from other sources. After screening, 11 studies fit the criteria for inclusion in the review (Fig. 1). These originated from the United States (six), Australia (three), the UK (one) and Kazakhstan (one) . Study designs were heterogeneous. Six studies utilised a relevant comparator group [9, 11, 19-22]. Of these, two were retrospective cohort studies of TB outbreaks $[9,11]$, two used routinely collected data $[19,21]$, one was a matched case-control study [20], and one a case series study that included a retrospective cohort study of contacts [22]. These six studies contributed seven effect estimates, as Davis et al [20] contributed two separate results. Of the remaining five studies four were descriptive outbreak reports/investigations [10, 23-25] and one was an analytic study [26] where the outcome of interest was having $\mathrm{TB}$ disease from which a specific strain of TB was isolated (as compared with having TB caused by a different strain) (Table 1).

\section{Risk of bias in studies which utilised a relevant comparator group}

Risk of bias assessments for each of the six studies which used a comparator group are presented in Table 2. All seven results (reported by the six studies) were judged to be at "Serious" risk of bias overall. These overall "Serious" judgments were largely due to the "Bias due to confounding' domain, 'Bias in selection of participants into the study' domain, and the 'Bias in measurement of outcomes' domain. Two studies were at "Serious" risk of bias due to the extent of missing data.

\section{Results of studies which utilised a relevant comparator group (Table 1)}

Cannabis use as a risk factor for latent TB

Four studies reported on the association between cannabis use and the risk of latent TB. Morano et al analysed

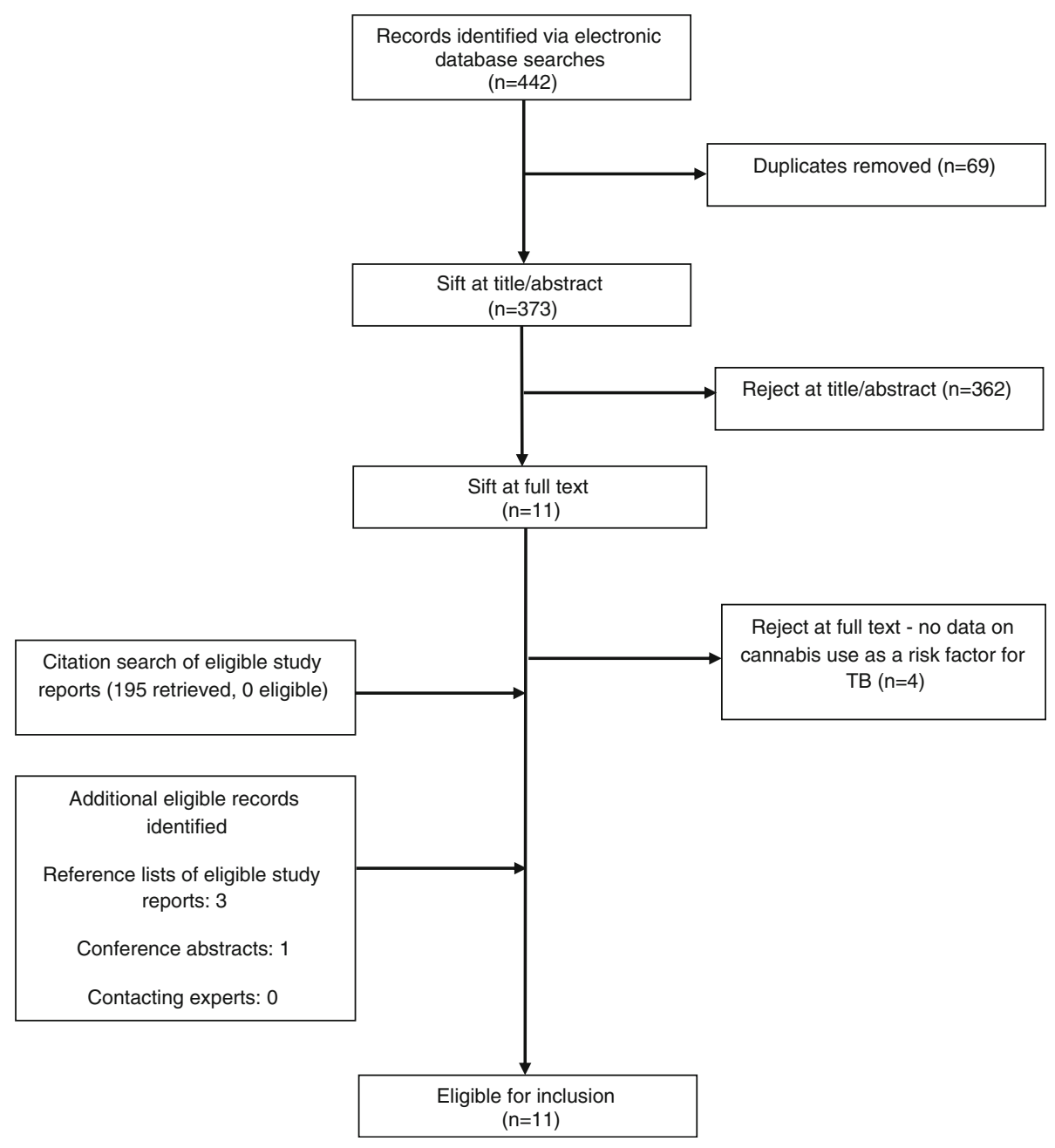

Fig. 1 Study selection flow chart 
Table 2 Risk of bias assessments

\begin{tabular}{|c|c|c|c|c|c|c|c|}
\hline \multirow[t]{2}{*}{ Author, year } & \multicolumn{6}{|c|}{ Domain-level judgments } & \multirow{2}{*}{$\begin{array}{l}\text { Overall } \\
\text { judgement }\end{array}$} \\
\hline & $\begin{array}{l}\text { Bias due to } \\
\text { confounding }\end{array}$ & $\begin{array}{l}\text { Bias in selection } \\
\text { of participants } \\
\text { into the study }\end{array}$ & $\begin{array}{l}\text { Bias in } \\
\text { classification } \\
\text { of exposures }\end{array}$ & $\begin{array}{l}\text { Bias due to } \\
\text { missing data }\end{array}$ & $\begin{array}{l}\text { Bias in } \\
\text { measurement } \\
\text { of outcomes }\end{array}$ & $\begin{array}{l}\text { Bias in selection } \\
\text { of the reported } \\
\text { result }\end{array}$ & \\
\hline Morano, 2014 [21] & Moderate & Moderate & Moderate & Serious & Moderate & Moderate & Serious \\
\hline Munckhof, 2003 [11] & Moderate & Serious & Moderate & Serious & Low & Moderate & Serious \\
\hline Thu, 2014 [22] & Serious & Serious & Moderate & No information & Low & Moderate & Serious \\
\hline Oeltmann, 2006 [9] & Serious & Serious & Moderate & No information & Low & Moderate & Serious \\
\hline Han, 2010 [19] & Moderate & Moderate & Moderate & No information & Serious & Moderate & Serious \\
\hline Davis, 2017 [20] & Moderate & Low & Moderate & No information & Serious & Moderate & Serious \\
\hline Davis, 2017 $[20]$ & Serious & Low & Moderate & No information & Serious & Moderate & Serious \\
\hline
\end{tabular}

aver used cannabis

${ }^{b}$ Cannabis use within last 90 days

data routinely collected by a mobile medical clinic and found cannabis use ('ever') to be associated with incident (but not prevalent) latent TB infection after adjustment for confounders (adjusted odds ratio [aOR]: 1.57; 95\% confidence interval [CI]:1.05-2.37) [21].

One retrospective cohort study [11] and one retrospective case series combined with a retrospective cohort study of contacts [22] assessed the association between sharing a cannabis bong with a TB case and the odds of having latent TB. In the cohort study of 149 identified contacts, 45 had shared a cannabis bong with a case. The aOR for latent $\mathrm{TB}$ in those who shared a bong with a pulmonary TB case vs. those who had not was 2.22 (95\% CI:0.96-5.17). In this cluster, cannabis smoking was frequently performed in closed rooms or cars. The authors noted that it was not possible to disaggregate relative contributions of sharing a bong and prolonged confinement in a shared airspace [11]. In the case series all three cases were young adults who reported regularly smoking cannabis through a bong. Of 111 contacts screened, 34 were positive for latent TB (positive TST), one of whom developed active TB. Contacts who shared a bong with an active TB case $(n=7)$ had a six-fold risk of a positive TST (OR 6.5, 95\% CI: $1.4-30.4, p=0.016$ ), though there was no adjustment for confounders [22].

One retrospective cohort study reviewed the association between being a member of a closely connected network of young men 'friends' who exhibited similar cannabis using behaviour [9]. There were 11 culture-confirmed cases ( 8 were sputum acid-fast bacilli (AFB) sputum smear positive), all of whom reported frequent 'hotboxing' (smoking cannabis in a confined space, such as a car, to maximise the effect [27]). The risk ratio of positive TST in the 'friends' contacts vs. other contacts was 2.8 (95\% CI: 1.3-6.0). Contacts with a past positive TST result were excluded from the analyses but no adjustment was made for confounding.

\section{Cannabis use as a risk factor for active TB disease}

Two studies reported on the association between cannabis use and having active TB disease. An analysis of routinely collected data found no evidence that cannabis use was associated with the risk of ever being diagnosed with TB disease; using those who had never used cannabis as the reference, the aORs were 0.79 (95\% CI:0.331.87) for those with $\leq 1$ year use, 0.72 (95\% CI:0.25-2.06) for those with 2-10 years use, and 0.73 (95\% CI:0.603.28) for those with $\geq 11$ years use [19]. A matched case-control study also demonstrated that after adjustment for confounding factors there was no statistical evidence of an association with ever having used cannabis and recent TB disease (aOR:1.64, 95\% CI:0.76-3.54, $p=0.210[20]$.

\section{Results of studies which did not utilise a relevant comparator group (Table 1) Cannabis use as a risk factor for latent TB}

Livengood et al conducted an investigation of contacts of a patient with culture and AFB sputum smear positive isoniazid-resistant TB [23]. Cannabis use was identified as the most important social risk factor for TB transmission - 100\% (14/14) of those contacts who used cannabis had a positive TST result. The authors report that the practice of 'hotboxing' undoubtedly contributed to TB transmission.

\section{Cannabis use as a risk factor for active TB disease}

Four studies reported on cannabis use as a risk factor for active TB disease. Merritt et al characterised a cluster of nine cases and two probable cases of pulmonary TB [24]. Cannabis use was identified in 67\% (6/9) of confirmed cases and both the suspected cases. Authors state that use of shared smoking equipment was not explored.

Sterling et al reported on a TB outbreak among a highly mobile population in the US. The index case had AFB smear positive pulmonary disease. Twenty outbreak 
cases were identified, $35 \%$ of whom $(n=7)$ reported cannabis use [10].

Evans et al reviewed the association between cannabis use and having a specific TB strain by examining the epidemiological characteristics of culture-positive TB cases with an indistinguishable MIRU-VNTR profile (the "Mercian" strain) [26]. Eleven of the 35 cases (31\%) with the Mercian strain reported cannabis use compared with 2/47 (4\%) with other TB strains (OR: 10.02, 95\% CI: $1.96-100.3, p<0.01)$.

Finally, McElroy et al investigated a cluster of 22 outbreak-associated TB patients (including 19 adults); $79 \%$ of the 19 active TB cases reported cannabis use, compared with $35 \%$ of a convenience sample of primary contacts (33\% of those TST positive and $36 \%$ of those TST negative). Sputum AFB status was reported for female cases $(n=9)$ only; seven had smear-positive cavitary pulmonary TB [25].

\section{Discussion}

We found 11 studies investigating the association between cannabis use and TB infection or disease. All studies were observational and most originated from high-income settings (10/11 studies). Six studies utilised a relevant comparator group. Four of these investigated the association between cannabis use and latent TB infection; all provided some evidence of an association, although only two of these had adjusted for confounders. The remaining two comparator studies investigated the association between cannabis use and active TB disease; neither found evidence of an association after adjusting for confounding. Five studies did not utilise a relevant comparator group; all indicated that TB outbreaks do occur among cannabis users but were not designed to test for an association.

Overall the quality of the evidence was weak and of insufficient quality to reliably quantify the risk. There was heterogeneity across populations studied and approaches to data analysis. Description of the exposure in terms of type and quantity, frequency, timing and method of cannabis use was largely inadequate. All six comparative studies were assessed as being at "Serious" risk of bias. Three of the seven results obtained from the six studies did not adjust for confounding. This may be indicative of the contexts in which studies were conducted - three emanated from outbreak investigations and the remainder utilised existing data sources not specifically designed to test this hypothesis. Where confounding variables were adjusted for these were heterogeneous across studies. Three results were assessed as being at "Serious" risk of 'bias in the measurement of outcomes', e.g. because investigators relied on self-reported TB status. In general, there was a lack of clear and sufficiently comprehensive information reported - e.g. for five of the seven results we could not assess 'bias due to missing data' as no relevant data was available. It is important that the poor quality of the available studies, with respect to addressing the review question, is borne in mind and care taken not to over interpret the data presented in this review.

The exposure and outcome measures used were not always appropriate for assessing an association between cannabis use and TB. For instance, several studies recorded the exposure as 'ever used' cannabis or the outcome as 'ever' having a diagnosis of TB. We felt it appropriate to include such studies in the review, particularly given the paucity of evidence but note that any association between cannabis and TB will likely differ depending on whether use is current or past and whether cannabis use preceded TB diagnosis. Additionally, studies lacked information on whether cannabis was used alone or with tobacco. Smoking tobacco is known to be associated with TB risk [15]. Practice varies globally - cannabis is commonly mixed with tobacco in Europe but is much more likely used alone in the US, while in Australia both practices are equally used [28]. Most studies in this review originated from the US (six), followed by Australia (three). These studies, particularly those from the US, and the two from Australia reporting on cannabis smoking via a water pipe, are therefore likely to provide some evidence, though not necessarily exclusively, relating to the specific effect of cannabis (rather than tobacco, or the combination of the two) on TB risk.

The aim of this review was to establish whether there is evidence of an association between cannabis use and TB acquisition, rather than to elicit the mechanisms by which any such association might operate. Indeed, with the evidence available it is not possible to disentangle the relative contributions of TB transmission being facilitated via close contact/shared air space (e.g. via 'hotboxing'), the spread of TB via contaminated equipment (e.g. via shared water pipes) or cannabis use as a more general social risk factor for TB, as compared with the potential biological or physiological mechanisms through which the act of inhaling cannabis may itself increase susceptibility to TB (e.g. through damage to the lungs).

The presence of AFB in the sputum is an important risk factor for onward transmission of TB [29, 30]. None of the studies in this review directly investigated whether sputum smear status influenced the association between cannabis use and TB. However, of the six studies which used a relevant comparator group, three reported that either all or most of the active TB cases were AFB smear positive. All of these three studies reported some evidence of an association between cannabis use and TB acquisition $[9,11,22]$.

\section{Strengths and limitations of the review process}

We conducted a comprehensive literature search, with no language or date restrictions in order to ensure, as 
far as possible, that we identified all relevant studies. Though not a limitation of the review process as such, we note that publication bias is a potential concern outbreak investigations are primarily purposed for disease control and many remain unpublished in any form, particularly if there are no unusual findings. Two authors independently assessed each study for eligibility, extracted the data and conducted the risk of bias assessments. This approach will have helped minimise both errors and potential biases in the review process.

ROBINS-I is a comprehensive published tool for assessing risk of bias in non-randomised studies. Since the tool is not specifically designed for assessing studies of exposures, we recognise that it may be limited in its ability to accurately classify the risk of bias in such studies. To help mitigate this we performed assessments at the domain level without answering the intra-domain signalling questions that are tailored to assessing intervention studies. We also used brief preliminary guidance for the forthcoming ROBINS-E tool tailored for studies of exposures. These approaches should reduce the potential for misclassification of bias judgements introduced by the inconsistency between study design and assessment tool. We do, however, recognise that any such risk of bias assessments may be open to subjective interpretation particularly where detailed information about study methodology is not reported, as was the case for several studies in this review.

\section{Conclusions}

The evidence for an association between cannabis use and TB is weak. We found a lack of studies on the topic, and those that are available are of poor quality. We found some evidence for an association between cannabis use and latent TB infection, but little evidence for an association with TB disease. Many of the studies we reviewed emanated directly from field investigations of TB clusters and outbreaks including contact tracing, with the aim to identify the source and transmission routes to prevent further spread. Consequently, these were not designed specifically to assess the association between cannabis use and TB and suffered from bias at the design, conduct and analysis stages. However, a possible association between cannabis use and TB has been indicated in several outbreak investigations and it cannot be excluded as a possible risk factor based on the current literature. Indeed this remains an important question to answer to inform future outbreak control measures, especially in light of potential increased cannabis use in the context of decriminalisation policies [31].

\section{Implications for practice and research}

The evidence base on cannabis use and TB needs to be strengthened. Opportunities exist in the context of wholegenome sequencing to more accurately distinguish cases that are part of a recent transmission cluster. The introduction of universal minimum standards for the reporting of infectious disease outbreaks could help improve the utility of published outbreak reports and must include accurate and comparable information on exposures, including, in this case, cannabis use practices, dose, frequency and timing. Efforts to facilitate observational epidemiological studies that include cannabis and other key exposures are needed and must include the collection and adjustment of key confounding factors. Studies designed to assess the independent association of cannabis use on TB infection and disease are ultimately required in order to specify the relative risk of this behaviour in the context of closely related risk factors such as close proximity and shared airspace.

\section{Additional files}

Additional file 1: Search strategies. (DOCX $13 \mathrm{~kb}$ )

Additional file 2: Guidance used to make domain level risk of bias judgements. (DOCX $18 \mathrm{~kb}$ )

\section{Abbreviations}

AFB: Acid-Fast Bacilli; aOR: Adjusted odds ratio; Cl: Confidence interval; OR: Odds ratio; TB: Tuberculosis; TST: Tuberculin Skin Test

\section{Acknowledgements}

We would like to thank Sarah Dawson (University of Bristol) for developing the search strategy and running the electronic database searches. We also recognise the contribution of Charlotte Prew (Public Health England) in developing and running a preliminary scoping search.

\section{Authors' contributions}

IO, CEF, CMC, SN and LA developed the review concept. CEF and CMC designed the review, screened studies for eligibility, extracted and analysed the data. LAM adapted the risk of bias tool for use in this review. CEF, CMC and LAM conducted the risk of bias assessments. CEF and CMC jointly drafted the manuscript. CEF, CMC, IO, SN, LA, LAM, CRB and MH contributed to the interpretation of the data and writing of the final manuscript and approved it for submission.

\section{Funding}

The research was funded by the National Institute for Health Research Health Protection Research Unit (NIHR HPRU) in Evaluation of Interventions at the University of Bristol in partnership with Public Health England (PHE). The views expressed are those of the author(s) and not necessarily those of the NHS, the NIHR, the Department of Health or Public Health England. The funding body had no role in the design of the study, collection, analysis, or interpretation of data, or in writing the manuscript.

\section{Availability of data and materials}

The data extracted and analysed are included in this published article and additional files.

Ethics approval and consent to participate Not applicable.

Consent for publication Not applicable.

Competing interests

The authors declare that they have no competing interests. 


\section{Publisher's Note}

Springer Nature remains neutral with regard to jurisdictional claims in published maps and institutional affiliations.

\section{Author details}

${ }^{1}$ Population Health Sciences, Bristol Medical School, University of Bristol, Bristol BS8 2PS, UK. ${ }^{2}$ National Institute for Health Research Health Protection Unit in Evaluation of Interventions, Bristol Medical School, University of Bristol, Bristol BS8 2PS, UK. ${ }^{3}$ Field Service South West, National Infection Service, Public Health England, Bristol BS1 6EH, UK. ${ }^{4}$ Field Service East Midlands, National Infection Service, Public Health England, Nottingham NG2 4LA, UK. ${ }^{5}$ Public Health England East Midlands, Public Health England Nottingham NG2 4LA, UK.

Received: 28 February 2019 Accepted: 7 June 2019

Published online: 27 July 2019

\section{References}

1. World Health Organization. Global Tuberculosis Report 2017. Geneva: World Health Organization; 2017.

2. European Centre for Disease Prevention and Control/WHO Regional Office for Europe. Tuberculosis surveillance and monitoring in Europe 2016. Stockholm, Sweden: European Centre for Disease Prevention and Control; 2016.

3. Fok A, Numata Y, Schulzer M, FitzGerald MJ. Risk factors for clustering of tuberculosis cases: a systematic review of population-based molecular epidemiology studies. Int J Tuberc Lung Dis. 2008;12(5):480-92.

4. Smith CM, Maguire H, Anderson C, Macdonald N, Hayward AC. Multiple large clusters of tuberculosis in London: a cross-sectional analysis of molecular and spatial data. ERJ Open Research. 2017;3(1):00098-2016.

5. Walker TM, Ip CL, Harrell RH, Evans JT, Kapatai G, Dedicoat MJ, Eyre DW Wilson DJ, Hawkey PM, Crook DW, et al. Whole-genome sequencing to delineate mycobacterium tuberculosis outbreaks: a retrospective observational study. Lancet Infect Dis. 2013;13(2):137-46.

6. European Monitoring Centre for Drugs and Drug Addiction. European drug report 2017: trends and developments. Luxembourg: Publications Office of the European Union; 2017.

7. Center for Behavioral Health Statistics and Quality. 2016 National Survey on Drug Use and Health: Detailed Tables. Rockville, MD: Substance Abuse and Mental Health Services Administration; 2017.

8. Australian Institute of Health and Welfare. National Drug Strategy Household Survey 2016: detailed findings. Canberra: Australian Institute of Health and Welfare; 2017.

9. Oeltmann JE, Oren E, Haddad MB, Lake L, Harrington TA, ljaz K, Narita M. Tuberculosis outbreak in marijuana users, Seattle, Washington, 2004. Emerg Infect Dis. 2006;12(7):1156-9.

10. Sterling TR, Thompson D, Stanley RL, McElroy PD, Madison A, Moore K, Ridzon R, Harrington S, Bishai WR, Chaisson RE, et al. A multi-state outbreak of tuberculosis among members of a highly mobile social network: implications for tuberculosis elimination. Int J Tuberc Lung Dis. 2000:4(11):1066-73.

11. Munckhof WJ, Konstantinos A, Wamsley M, Mortlock M, Gilpin C. A cluster of tuberculosis associated with use of a marijuana water pipe. Int J Tuberc Lung Dis. 2003;7(9):860-5.

12. Perlman DC, Perkins MP, Paone D, Kochems L, Salomon N, Friedmann P, Des Jarlais DC. "Shotgunning" as an illicit drug smoking practice. J Subst Abus Treat. 1997;14(1):3-9.

13. Joshi M, Joshi A, Bartter T. Marijuana and lung diseases. Curr Opin Pulm Med. 2014:20(2):173-9.

14. Den Boon S, Van Lill SWP, Borgdorff MW, Verver S, Bateman ED, Lombard CJ, Enarson DA, Beyers N. Association between smoking and tuberculosis infection: a population survey in a high tuberculosis incidence area. Thorax. 2005;60(7):555-7.

15. Bates MN, Khalakdina A, Pai M, Chang L, Lessa F, Smith KR. Risk of tuberculosis from exposure to tobacco smoke: a systematic review and meta-analysis. Arch Intern Med. 2007;167(4):335-42.

16. Moher D, Liberati A, Tetzlaff J, Altman DG. Preferred reporting items for systematic reviews and meta-analyses: the PRISMA statement. BMJ. 2009;339.

17. Sterne JA, Hernán MA, Reeves BC, Savović J, Berkman ND, Viswanathan M, Henry D, Altman DG, Ansari MT, Boutron I, et al. ROBINS-I: a tool for assessing risk of bias in non-randomised studies of interventions. BMJ. 2016; 355:i4919.

18. The ROBINS-E tool (Risk of Bias in non-randomized studies - of exposures) (preliminary tool) https://www.bristol.ac.uk/population-health-sciences/ centres/cresyda/barr/riskofbias/robins-e/. Accessed May 2018.

19. Han B, Gfroerer JC, Colliver JD. Associations between duration of illicit drug use and health conditions: results from the 2005-2007 national surveys on drug use and health. Ann Epidemiol. 2010;20(4):289-97.

20. Davis A, Terlikbayeva A, Aifah A, Hermosilla S, Zhumadilov Z, Berikova E, Rakhimova S, Primbetova S, Darisheva M, Schluger N, et al. Risks for tuberculosis in Kazakhstan: implications for prevention. Int J Tuberc Lung Dis. 2017;21(1):86-92.

21. Morano JP, Zelenev A, Walton MR, Bruce RD, Altice FL. Latent tuberculosis infection screening in foreign-born populations: a successful mobile clinic outreach model. Am J Public Health. 2014;104(8):1508-15.

22. Thu K, Hayes M, Miles S, Tierney L, Foy A. Marijuana 'bong' smoking and tuberculosis. Intern Med J. 2013:43(4):456-8.

23. Livengood JR, Sigler T, Foster L, Bobst J, Snider D Jr. Isoniazid-resistant tuberculosis. JAMA. 1985;253:2847-9.

24. Merritt TD, Sintchenko V, Jelfs P, Worthing M, Robinson B, Durrheim DN Gilbert GL. An outbreak of pulmonary tuberculosis in young Australians. Med J Aust. 2007:186(5):240.

25. McElroy PD, Rothenberg RB, Varghese R, Woodruff R, Minns GO, Muth SQ, Lambert LA, Ridzon R. A network-informed approach to investigating a tuberculosis outbreak: implications for enhancing contact investigations. Int J Tuberc Lung Dis. 2003:7(12):486-93.

26. Evans JT, Serafino Wani RL, Anderson L, Gibson AL, Smith EG, Wood A, Olowokure B, Abubakar I, Mann JS, Gardiner S, et al. A geographicallyrestricted but prevalent mycobacterium tuberculosis strain identified in the west midlands region of the UK between 1995 and 2008. PLoS One. 2011; 6(3):e17930

27. Lutchmansingh D, Pawar L, Savici D. Legalizing Cannabis: a physician's primer on the pulmonary effects of marijuana. Current Respiratory Care Reports. 2014;3(4):200-5.

28. Winstock AR, Barratt MJ, Maier LJ, Ferris JA. Global drug survey 2018 - key findings report. Global Drug Survey; 2018.

29. Sepkowitz K. How contagious is tuberculosis? Clin Infect Dis. 1996;23(5): 954-62.

30. Narasimhan P, Wood J, Macintyre C, Mathai D. Risk factors for tuberculosis. Pulm Med. 2013:828939.

31. Eastwood N, Fox E, Rosmarin A. Quiet revolution: drug decriminalisation across the globe. London: Release - Drugs, The Law and Human Rights; 2016.

Ready to submit your research? Choose BMC and benefit from:

- fast, convenient online submission

- thorough peer review by experienced researchers in your field

- rapid publication on acceptance

- support for research data, including large and complex data types

- gold Open Access which fosters wider collaboration and increased citations

- maximum visibility for your research: over $100 \mathrm{M}$ website views per year

At BMC, research is always in progress.

Learn more biomedcentral.com/submissions 